\title{
Flow diversion treatment Intra-aneurismal blood flow velocity and WSS reduction are parameters to predict aneurysm thrombosis
}

\section{Journal Article}

\section{Author(s):}

Kulcsár, Zsolt; Augsburger, Luca; Reymond, Philippe; Pereira, Vitor M.; Hirsch, Sven; Mallik, Ajit S.; Millar, John; Wetzel, Stephan G.; Wanke, Isabel; Rüfenacht, Daniel A.

\section{Publication date:}

2012-10

\section{Permanent link:}

https://doi.org/10.3929/ethz-b-000055546

\section{Rights / license:}

In Copyright - Non-Commercial Use Permitted

\section{Originally published in:}

Acta Neurochirurgica 154(10), https://doi.org/10.1007/s00701-012-1482-2 


\title{
Flow diversion treatment: intra-aneurismal blood flow velocity and WSS reduction are parameters to predict aneurysm thrombosis
}

\author{
Zsolt Kulcsár • Luca Augsburger • Philippe Reymond • \\ Vitor M. Pereira • Sven Hirsch • Ajit S. Mallik • \\ John Millar • Stephan G. Wetzel • Isabel Wanke • \\ Daniel A. Rüfenacht
}

Received: 30 May 2012 / Accepted: 10 August 2012 / Published online: 29 August 2012

(C) Springer-Verlag 2012

\begin{abstract}
Background To evaluate the haemodynamic changes induced by flow diversion treatment in cerebral aneurysms, resulting in thrombosis or persisting aneurysm patency over time.

Method Eight patients with aneurysms at the paraophthalmic segment of the internal carotid artery were treated by flow diversion only. The clinical follow-up ranged between 6 days and 12 months. Computational fluid dynamics (CFD) analysis of pre- and post-treatment conditions was performed in all cases. True geometric models of the flow diverter were created and placed over the neck of the aneurysms by using a virtual stent-deployment technique, and the device was simulated as a true physical barrier. Pre- and post-treatment haemodynamics were compared, including
\end{abstract}

\footnotetext{
Z. Kulcsár $(\bowtie) \cdot$ S. G. Wetzel · I. Wanke • D. A. Rüfenacht Department of Neuroradiology, Swiss Neuro Institute, Hirslanden Clinic,

Witellikerstr. 40,

8032 Zurich, Switzerland

e-mail: kulcsarzsolt22@gmail.com

Z. Kulcsár • A. S. Mallik • I. Wanke • D. A. Rüfenacht Center of Applied Biotechnology and Molecular Medicine, University of Zurich,

Zurich, Switzerland

L. Augsburger

StrokeLab Inc.,

Geneva, Switzerland

P. Reymond

Federal Institute of Technology,

Lausanne, Switzerland
}

mean and maximal velocities, wall-shear stress (WSS) and intra-aneurysmal flow patterns. The CFD study results were then correlated to angiographic follow-up studies.

Results Mean intra-aneurysmal flow velocities and WSS were significantly reduced in all aneurysms. Changes in flow patterns were recorded in only one case. Seven of eight aneurysms showed complete occlusion during the followup. One aneurysm remaining patent after 1 year showed no change in flow patterns. One aneurysm rupturing 5 days after treatment showed also no change in flow pattern, and no change in the maximal inflow velocity.

Conclusions Relative flow velocity and WSS reduction in and of itself may result in aneurysm thrombosis in the majority of cases. Flow reductions under aneurysm-specific thresholds may, however, be the reason why some

\footnotetext{
V. M. Pereira

Department of Neuroradiology, Geneva University Hospital, Geneva, Switzerland

S. Hirsch

Computer Vision Laboratory,

Swiss Federal Institute of Technology,

Zurich, Switzerland

J. Millar

Department of Neuroradiology, Wessex Neurological Centre, Southampton General Hospital,

Southampton, UK

I. Wanke

Department of Diagnostic and Interventional Radiology and Neuroradiology, University Hospital of Essen,

Essen, Germany
} 
aneurysms remain completely or partially patent after flow diversion.

Keywords Flow diversion $\cdot$ Flow diverter $\cdot$ Cerebral aneurysm · Computational fluid dynamics · Wall shear stress · Thrombosis

\section{Introduction}

Flow diversion technology has already shown great promise in therapeutic success and outcomes, especially in the treatment of aneurysms considered otherwise difficult or by parent vessel reconstruction not possible to treat $[3,9,13]$. The theoretical background for this technology relies on the fact that these devices induce changes in intra-aneurysmal haemodynamics, which result in progressive thrombosis, occlusion, reverse remodelling and healing of the parent vessel and aneurysm. But since the location, morphology and size of aneurysms are all different, the induced haemodynamic effect is also expected to alter in each case.

The use of flow diverters (FDs) very seldomly provides immediate thrombosis and occlusion of the aneurysm. With modification of intra-aneurysmal flow, and with patients under antiplatelet therapy, the healing process usually takes time, from days to months. Persisting patency also became well known to practitioners. Most importantly, it is still unknown how much flow reduction is needed to induce progressive aneurysm thrombosis, without leaving an aneurysm unprotected for a longer time or even exposing it to an increased risk of rupture. To date there are no reliable measures to predict the dynamics of aneurysm thrombosis. According to this, the use of flow diverters in clinical practice still differs from centre to centre. One of the major differences relies on the number of overlapping layers used for the treatment of a single aneurysm. In some centres, when possible only a single device is used, with the hope that, irrelevant to the angiographically detectable intra-aneurysmal flow changes, thrombosis will occur over time. In other centres, more overlapping layers are deployed until a subjectively "acceptable" slow down of flow and stagnation of contrast material is appreciated.

Our purpose was hence to understand the haemodynamic changes induced in the aneurysms by a single layer flow diverter, and to correlate this with clinical follow-up data, in order to understand which haemodynamic parameter changes are needed to induce aneurysm occlusion. To reproduce the changes in haemodynamic conditions due to the FD, we performed flow simulations in a $3 \mathrm{D}$ model of the diseased vessel.

\section{Materials and methods}

Eight patients were retrospectively selected for this study. The most important selection criterion was para-ophthalmic location, as this is the most frequent location for FD treatment in the clinical practice. Having all aneurysms from the same location, the differences due to anatomical locationrelated haemodynamics were hence reduced. For consistency purposes, only patients treated with the same brand of FD device, the Silk (Balt International, Montmorency, France), were included in this analysis. Choosing flow diversion as treatment modality was decided on an individual basis, taking into consideration the achievable short- and longterm outcome results with conventional reconstructive endovascular methods (coiling alone or balloon or stent-assisted coiling). The FD device was in all cases placed in only one layer, without additional use of coils or other embolic material. Patients were prepared with aspirin and clopidogrel prior to procedure, and the double antiplatelet regime was continued for 2-3 months post-procedure, whereas aspirin was continued during the whole follow-up period. A threedimensional rotational angiography (3DRA) was obtained in all patients prior to FD implantation. From this 3D dataset the concerned vascular segment was extracted and used for computational flow dynamics analysis. A database containing patients' demographics, aneurysm location, dome and neck size and aspect ratio (AR), and aneurysm flow characteristics, as evaluated by the computerised flow dynamic analysis, before and after FD implantation (mean and maximal intra-aneurysmal flow velocity, mean and maximal intra-aneurysmal wall-shear stress (WSS), intra-aneurysmal flow patterns) was created.

The patients were followed for 6-12 months; the aneurysm occlusion status was verified by digital subtraction angiography (DSA), by CT angiography (CTA) or MR angiography (MRA).

Vascular and stent models

We reproduced the geometric domain and the position of the FD to perform flow simulation with and without the device. We constructed a virtual model of the FD, resembling the geometry of the actual Silk device - a braided stent composed of 48 wires, 40 wires having a diameter of $30 \mu \mathrm{m}$ and eight having a diameter of $50 \mu \mathrm{m}$. According to calculations, the porosity and pore density of an unconstrained device are $61 \%$ and 21 pores $/ \mathrm{mm}^{2}$ respectively. Rhinoceros 4.0 (Seattle, WA, USA) software was used to place the virtual stent inside the vessel model by bending and fitting the geometrical stent model to the aneurysm neck and parent artery wall. The porosity of the deformed stent was used in the simulations. To reduce computational efforts only the stent struts at the aneurismal neck were regarded, whereas 
the struts lodged against the artery wall in other areas were excluded from the model.

After segmentation, the meshing of the domain was performed with ICEM CFD 11.0 (Ansys, Canonsburg, PA, USA). As the dimensions of the FD struts are small compared with the vessel dimension, we preferred an adaptive mesh approach. Mesh smoothing was applied using Ansys inherent smoothing routines. Blood flow was simulated using Fluent 6.0 (Ansys, Canonsburg, PA, 15317) software. Blood was regarded as an incompressible Newtonian fluid with a density of $1.06 \mathrm{~g} / \mathrm{cm}^{3}$ and a viscosity of $4 \mathrm{mPa} \cdot \mathrm{s}$. A fully developed flow, as characterised by the Womersley solution, was imposed as boundary condition at the inflow plane. The vessel wall is assumed to be rigid with no slip boundary condition, the outlets were set to a zero pressure condition.

As patient-specific blood flow measurements were not available for the patients analyzed, we set flow rate profiles using a generic one-dimensional (1-D) physiological model [11]. The pulse follows a flow rate between $2.1 \mathrm{ml} / \mathrm{s}$ and $11.1 \mathrm{ml} / \mathrm{s}$ (average flow $4.8 \mathrm{ml} / \mathrm{s}$ over full cardiac cycle) with a velocity between $15.9 \mathrm{~cm} / \mathrm{s}$ and $84.1 \mathrm{~cm} / \mathrm{s}$ (average velocity $36.4 \mathrm{~cm} / \mathrm{s}$ over full cardiac cycle). This results in a Reynolds number of between 173 and 914 (average 396), where the flow is mainly laminar. The Womersley number of 2.6 characterises a transitional regime with both inertial effect and viscous effect. The transient flow situation was computed with a time step of $0.01 \mathrm{~s}$ for a cardiac cycle period of $T=0.8 \mathrm{~s}$. In each simulation, three cardiac cycles were computed and only the last cardiac cycle was considered for the analysis.

Definition of shear-driven and inertia-driven flow The exchange of blood between the aneurysm and the parent artery and the energy deposition depend on the flow conditions. For shear-driven flow, the aneurysm flow is created by friction from the parent artery flow. The flow energy into the aneurysm is low and a low exchange of blood between the parent artery and the aneurysm cavity is observed. For inertia-driven flow, the parent artery flow points directly into the aneurysm. The flow hits the aneurysm dome directly, creating intense fluidic exchange between the parent artery and the aneurysm.

\section{Results}

Aneurysm characteristics and treatment details

The mean age of the patients was $53 \pm 10$ years, and there was only one male. All aneurysms were previously unruptured. The mean of the maximal aneurysm diameter was $10 \mathrm{~mm}$, with a range of $5.5-21.5 \mathrm{~mm}$, and the mean neck size was $4.5 \mathrm{~mm}$ (range, 3-6 mm). The mean AR value was 2.2 (range, 0.9-4.5). Four aneurysms were regular in shape, and of four irregular shaped aneurysms, two presented with small blebs on the surface.

All aneurysms were treated with one Silk FD device only. One patient experienced a delayed rupture of the aneurysm 5 days after treatment, causing her death. One patient stopped clopidogrel 4 weeks after treatment, resulting in embolic occlusion of the central retinal artery. This paraophthalmic aneurysm was at this time completely thrombosed. No further post-procedural complications occurred. The results are summarised in Table 1.

Occlusion rates after treatment

The follow-up imaging of patients was performed at different intervals. Image documentation of aneurysm occlusion was obtained within a range from 1 to 10 months. One of the aneurysms showed complete patency and unchanged morphology at 12 months follow-up, with the patient reduced to single antiplatelet therapy with aspirin only after 3 months of FD implantation. The aneurysm rupturing at 5 days showed near to complete thrombosis, with the patient being under double antiplatelet therapy.

Intra-aneurysmal haemodynamics before and after FD placement

In the unstented state, the computational fluid dynamics (CFD) simulations of the aneurysms have shown an inertia driven flow in three of the eight cases, and a shear-driven flow in five aneurysms. All aneurysms showed one intraaneurysmal vortex, except for an aneurysm with two lobes, where one vortex was present in each lobe. The change in flow is characterised as a relative change in velocity and wall shear stress. The FD induced important reduction of mean intra-aneurysmal flow velocities (44\% reduction, $12.3 \pm 5.3 \mathrm{~cm} / \mathrm{s}$ pre-stent vs $6.9 \pm 4.2 \mathrm{~cm} / \mathrm{s}$ post-stent for the mean inflow velocities). The maximal inflow velocity was slightly less, but still highly reduced (29\% reduction, $69.1 \pm$ $16 \mathrm{~cm} / \mathrm{s}$ vs $50 \pm 24 \mathrm{~cm} / \mathrm{s}$ ). The mean and maximal intraaneurysmal WSS was also significantly reduced (59\% reduction, $2.9 \pm 1.8 \mathrm{~Pa}$ vs $1.3 \pm 1.1 \mathrm{~Pa}$; and $58 \%$ reduction, $70.6 \pm 68.8 \mathrm{~Pa}$ vs $28 \pm 31 \mathrm{~Pa}$, respectively).

By dividing aneurysms according to size into two groups with a cutoff value of $10 \mathrm{~mm}$ (two aneurysms $>10 \mathrm{~mm}$, six aneurysms $<10 \mathrm{~mm}$ ), the mean and maximal intraaneurysmal flow velocities were remarkably different between the two groups, both before and after FD placement (mean velocity before/after FD: $6 \mathrm{~cm} / \mathrm{s} / 3 \mathrm{~cm} / \mathrm{s}$ for the $>10 \mathrm{~mm}$ aneurysms vs $14.5 \mathrm{~cm} / \mathrm{s} / 8 \mathrm{~cm} / \mathrm{s}$ for the $<10 \mathrm{~mm}$ aneurysms), although the rate of change was very similar (45 \% vs $42 \%$ ). Same applied for the mean WSS 
Table 1 Patient and aneurysm characteristics

Flow changes

\begin{tabular}{|c|c|c|c|c|c|c|c|c|c|c|c|c|}
\hline \multirow[b]{2}{*}{ No. } & \multirow[b]{2}{*}{ Age } & \multirow[b]{2}{*}{ Gender } & \multicolumn{3}{|l|}{ Aneurysm } & \multicolumn{2}{|c|}{ Mean/max velocity $(\mathrm{m} / \mathrm{s})$} & \multirow[b]{2}{*}{$\begin{array}{l}\text { Change } \\
(\%)\end{array}$} & \multicolumn{2}{|c|}{ Mean/max WSS (Pa) } & \multirow[b]{2}{*}{$\begin{array}{l}\text { Change } \\
(\%)\end{array}$} & \multirow[b]{2}{*}{ Outcome } \\
\hline & & & Location & $\begin{array}{l}\text { Maximal diameter } \\
(\mathrm{mm})\end{array}$ & $\mathrm{AR}$ & Without FD & With FD & & $\begin{array}{l}\text { Without } \\
\text { FD }\end{array}$ & $\begin{array}{l}\text { With } \\
\text { FD }\end{array}$ & & \\
\hline 1 & 57 & $\mathrm{~F}$ & R ICA & 5.8 & 1.1 & $0.16 / 0.77$ & $0.13 / 0.67$ & $19 / 13$ & $6.05 / 166$ & $3.4 / 43$ & $44 / 74$ & Occlusion \\
\hline 2 & 55 & $\mathrm{~F}$ & L ICA & 6.1 & 2.0 & $0.07 / 0.38$ & $0.04 / 0.27$ & $39 / 29$ & $1.2 / 13$ & $0.6 / 5.3$ & $50 / 59$ & Occlusion \\
\hline 3 & 59 & $\mathrm{~F}$ & R ICA & 7.2 & 1.3 & $0.13 / 0.7$ & $0.12 / 0.62$ & $10 / 12$ & $4.1 / 167$ & $2.2 / 34$ & $46 / 80$ & Occlusion \\
\hline 4 & 71 & $\mathrm{~F}$ & L ICA & 20.3 & 4.5 & $0.07 / 0.63$ & $0.02 / 0.32$ & $72 / 49$ & $0.79 / 16$ & $0.2 / 5$ & $73 / 69$ & Occlusion \\
\hline 5 & 54 & $\mathrm{~F}$ & R ICA & 8.3 & 2.0 & $0.16 / 0.65$ & $0.09 / 0.45$ & $45 / 28$ & $2.57 / 21$ & $1.3 / 15$ & $48 / 32$ & Occlusion \\
\hline 6 & 45 & $\mathrm{~F}$ & R ICA & 21.5 & 3.6 & $0.05 / 00.97$ & $0.04 / 0.97$ & $20 / 0$ & $1.24 / 121$ & $0.5 / 97$ & $60 / 20$ & Rupture \\
\hline 7 & 37 & M & R ICA & 6.1 & 0.9 & $0.15 / 0.69$ & $0.03 / 0.29$ & $80 / 58$ & $3.28 / 20$ & $0.6 / 5.5$ & $81 / 72$ & Occlusion \\
\hline 8 & 45 & $\mathrm{~F}$ & L ICA & 5.6 & 1.3 & $0.2 / 0.74$ & $0.08 / 0.39$ & $60 / 47$ & $4.36 / 41$ & $1.4 / 16$ & $68 / 60$ & Persisting patency \\
\hline
\end{tabular}

(1 $\mathrm{Pa} / 0.2 \mathrm{~Pa}$ vs $3.6 \mathrm{~Pa} / 1.6 \mathrm{~Pa}$ respectively). The large aneurysms showed therefore a much slower intraaneurysmal flow and lower WSS.

Flow pattern changes

From the eight aneurysms, only one showed an assessable change in flow pattern, in terms of changed inflow profiles and flow streamlines (Fig. 1). In the other seven aneurysms, the intra-aneurysmal flow streamlines did not change after the placement of the FD, showing only reductions of the flow velocity and of the WSS. The aneurysm with changed flow patterns presented originally a shear driven flow, and showed complete occlusion during the follow-up.

Aneurysm with delayed rupture after treatment

The irregular shaped, 21.5-mm maximal diameter aneurysm (case no. 6) had an aspect ratio (AR) of 3.6. The aneurysm presented an inertia-driven flow, with a very high maximal inflow velocity of $97 \mathrm{~cm} / \mathrm{s}$, and with very low mean intra-aneurysmal flow velocities of $5 \mathrm{~cm} / \mathrm{s}$. The maximal WSS corresponding to the inflow zone area was also extremely high, $121 \mathrm{~Pa}$, as assessed by CFD simulation. In this case, the FD showed no effect on the intra-aneurysmal flow pattern. The maximal inflow velocity did not change after FD placement, and the maximal WSS at the level of the inflow area showed also only a $20 \%$ decrease and persisted at the barely high level of $97 \mathrm{~Pa}$. Post-treatment, the mean intra-aneurysmal WSS was highly reduced (with $44 \%$ ). Five days after FD placement the aneurysm ruptured, causing a massive subarachnoid haemorrhage. Both antiplatelet agents were stopped at this moment. A CTA performed 1 day later demonstrated complete occlusion of the aneurysm. There was one similar size (dome size, $20 \mathrm{~mm}$; AR, 4.5) and morphology aneurysm (case no. 4) in the database. In this case both the maximal inflow velocity and mean intra-aneurysmal velocity were highly reduced after FD placement (with $49 \%$ and $72 \%$ respectively), accompanied by an important reduction in the mean WSS (73\%). At the follow-up after 8 months, this aneurysm was completely thrombosed.

Aneurysm remaining patent after 1 year of treatment

This regular, broad based, 5.5-mm aneurysm (case no. 8), with an AR of 1.3 showed complete patency after 1 year, despite cessation of clopidogrel treatment 9 months before the last follow-up DSA. The aneurysm presented a sheardriven flow. The maximal and mean flow velocities were importantly reduced by the FD (74 to $39 \mathrm{~cm} / \mathrm{s}, 47 \%$ reduction, and 20 vs $8 \mathrm{~cm} / \mathrm{s}, 60 \%$ reduction respectively). The maximal and mean intra-aneurysmal WSS also showed major reduction after treatment (41 vs $16 \mathrm{~Pa}, 60 \%$ reduction, and 4.3 vs $1.4 \mathrm{~Pa}, 68 \%$ reduction respectively). In this case the FD showed no effect on the intra-aneurysmal flow patterns (Fig. 2). There was one aneurysm with similar size and location in the database (5.6 mm dome size; AR, 1.1), but with slightly different morphology. The reductions in maximal and mean flow velocities were less important in this case compared with the previous one $(77$ to $67 \mathrm{~cm} / \mathrm{s}$, $13 \%$ reduction, and 16 to $13 \mathrm{~cm} / \mathrm{s}, 19 \%$ reduction respectively). The WSS reductions were similar (mean WSS 6.1 to $3.4 \mathrm{~Pa}, 44 \%$ reduction), although the resulting WSS after FD placement was in magnitude 2.4-times higher in the nonoccluded aneurysm. Concerning flow pattern changes, this aneurysm showed an assessable difference in flow pattern before and after treatment. 

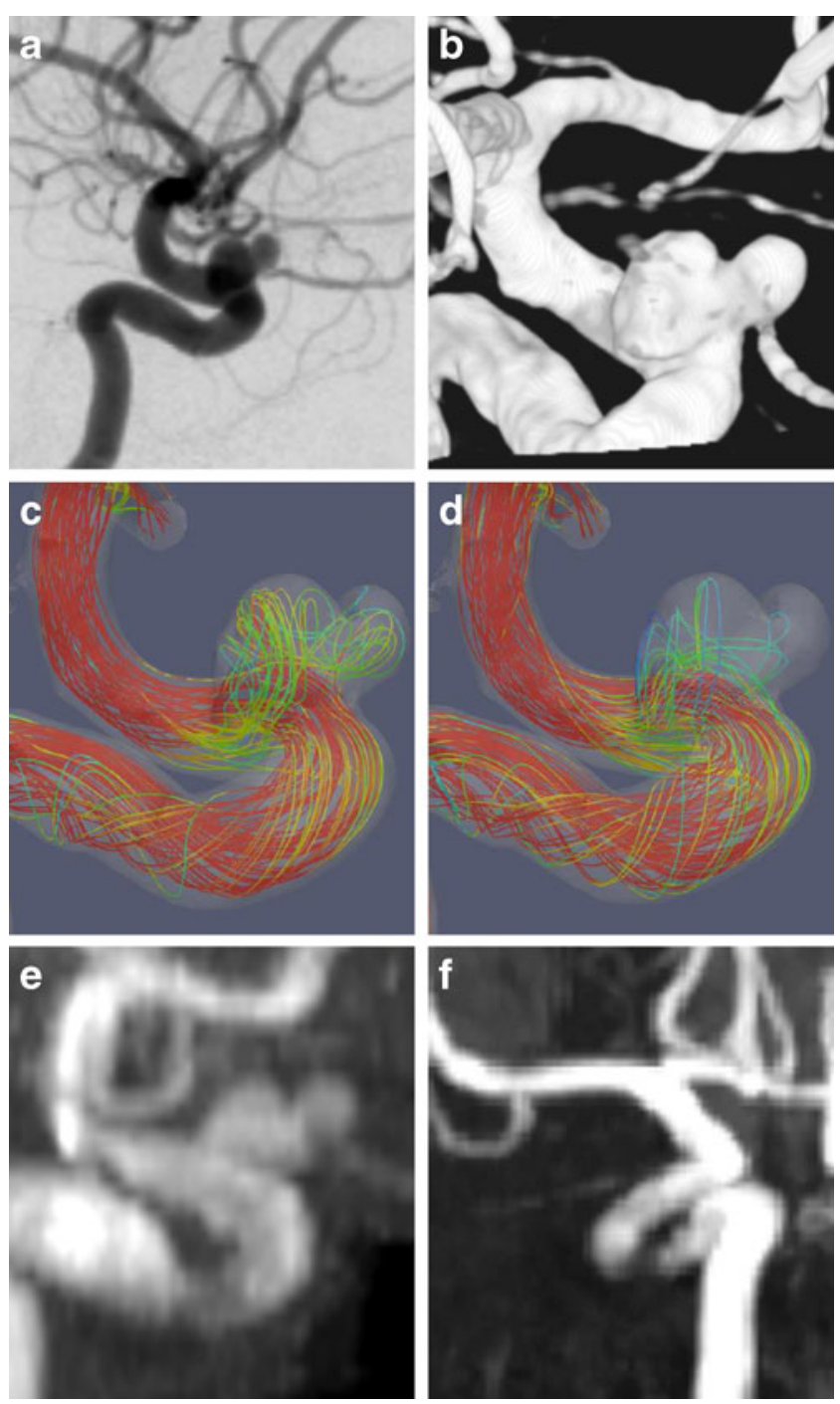

Fig. 1 Irregular para-ophthalmic aneurysm of the right ICA, with the ophthalmic artery arising from the aneurysm sac, DSA lateral view (a) and 3DRA reconstruction (b). CFD simulation of flow streamlines demonstrates an important change of flow pattern besides flow velocity reduction [before (c) and after FD placement (d)]. Time-of-flight (TOF) MRA $24 \mathrm{~h}$ after treatment demonstrating full patency of the aneurysm (e), and resorption (completed reverse remodelling) at the 9 months' follow-up (f)

\section{Discussion}

In this series of para-ophthalmic, originally non-ruptured aneurysms of the internal carotid artery treated by a singlelayer, braided, low-porosity, high-pore-density FD device, the effect of flow diversion on intra-aneurysmal flow was analysed and correlated with clinical outcome in terms of aneurysm occlusion. The FD induced an important reduction of the intra-aneurysmal flow velocities and the WSS in general, independent of aneurysm size. The flow pattern was changed in only one of eight aneurysms. Six of eight aneurysms showed a complete occlusion during the followup, while one aneurysm ruptured 5 days after treatment, and
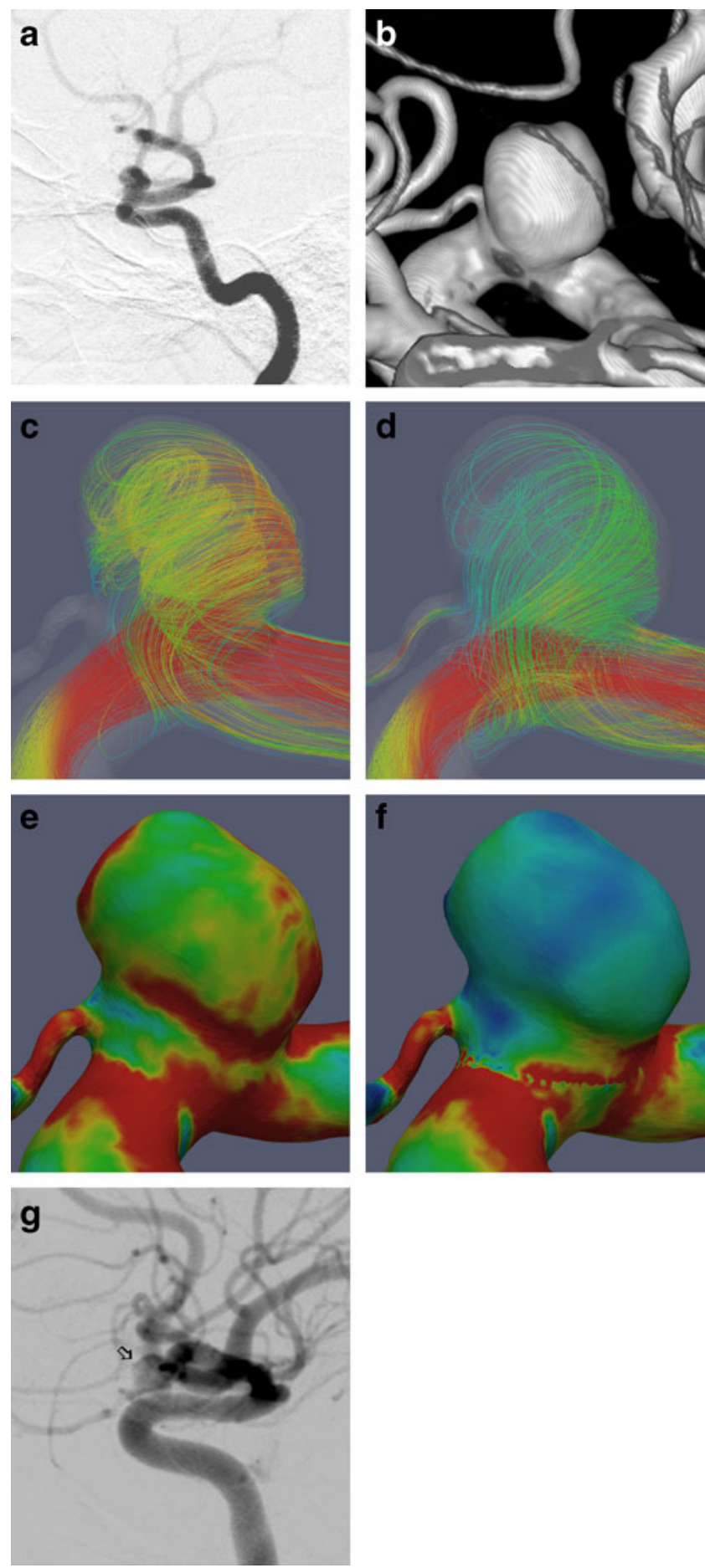

Fig. 2 Para-ophthalmic aneurysm of the left ICA, with the ophthalmic artery arising from the neck of the aneurysm, DSA lateral view (a) and 3DRA reconstruction (b). CFD simulation of flow streamlines before (c) and after (d), and WSS distribution before (e) and after (f) virtual placement of the FD over the aneurysm neck. Although the flow velocities and the WSS are considerably reduced, the flow pattern remained unchanged. The aneurysm remained patent 1 year after treatment, as shown by the lateral view DSA of the left ICA (g)

one aneurysm remained still unchanged at 1 year after device implantation. Having included only aneurysms at 
para-ophthalmic location, these results may not apply to aneurysms at other points of the circle of Willis.

FDs provided a very important endovascular tool for the reconstructive treatment of difficult and high recurrence rate aneurysms. Pre-clinical and clinical series have shown that these devices induce thrombosis of the aneurysm, followed by sealing of the luminal surface by a layer of neointimal tissue, which will consequently lead to cicatrisation and reverse remodelling of the aneurysm. The process of progressive thrombosis and aneurysm occlusion probably depends on several factors, but most importantly on the induced intra-aneurysmal flow changes. This is related to the size, location and morphological characteristics of the aneurysm, the number of FDs deployed over the neck (more layers may have a more pronounced flow diverting effect) and the deployment technique, which may have major influence on the FD porosity. On the other hand, the aneurysm thrombosis is probably also related to the ongoing antiplatelet therapy and the patient's personal haemostatic profile, although the impact of antiplatelet therapies on intraaneurysmal clot development is barely known. The process is seldom predictable, which may raise two major concerns. First, certain aneurysms treated with flow diversion alone seem to be not protected from rupture during the phase of thrombosis [8]. Second, some aneurysms remain patent and show no or only partial thrombosis during months or even years after treatment $[3,7,9,10,13]$. This may cause uncertainty for both the physician and patient, and on the other hand limit the re-treatment options (no more intrasaccular access through the device possible due to the high mesh density of the device).

In this series, the Silk FD device was simulated based on its real geometrical properties as true physical barrier over the neck of the aneurysm, as previously described [2]. In accordance with published small case studies [2, 4], our results have shown that the most important effect of the device is measurable in the relative reduction of intraaneurysmal flow velocities and WSS. The maximal inflow velocities were less reduced than the mean intra-aneurysmal flow velocities in general. The device showed a more important effect on reduction of mean and maximal intraaneurysmal WSS. Both these results suggest a real slowdown of intra-aneurysmal flow and an increased intraaneurysmal flow residence time compared with pretreatment status. The results are in concordance with the animal studies [12] and clinical experience, showing that FDs increase the contrast material washout time of the aneurysm. It is well known that slow flow and consequent low WSS are important factors for inducing thrombosis [1], which correlates with the clinical effect of flow diversion.

It is important to note that, as expected, large aneurysms showed a slower intra-aneurysmal flow and lower WSS than the small $(<10 \mathrm{~mm})$ ones. The mean flow velocities and the mean WSS after FD treatment in the small group were still higher than in the large group before treatment. Although the low number of cases does not allow for a real comparison, this strongly suggests that the induced relative change in the intra-aneurysmal flow profile will lead the process of thrombosis, and not the fair value of velocity and WSS. All aneurysms may therefore present specific threshold values of flow and WSS, where progressive thrombosis will be initiated.

By analysing the flow dynamics of the aneurysm with delayed rupture, the most important difference in comparison with the others was the aneurysm size and morphology, of and the lack of reduction of the maximal inflow velocity. This was the largest aneurysm from the series with a maximal diameter of $21.5 \mathrm{~mm}$, with an AR of 3.6. Both these parameters were already indirectly suggestive of aneurysm wall vulnerability and of an elevated risk of rupture $[14,15]$. Probably due to the further reduction, an already very slow mean flow velocity and WSS, the aneurysm demonstrated a quick thrombosis after treatment. Due to the quickly developed large clot volume, the aneurysm wall might be exposed to thrombus-induced autolytic activities and may be overwhelmed by the large destructive enzymatic overload, leading to structural destruction of the aneurysm wall, as previously described $[5,6,8]$. On the other hand, the maximal and otherwise very high inflow velocity was practically unchanged by the FD device, as measured by CFD, and the intra-aneurysmal flow patterns and the inflow zone also remained unchanged. We may hence hypothesise that the thrombus could not conquer this territory due to the persisting very high flow velocity in this region. This persisting inflow jet could then act as a dissector in between the already formed destructive thrombus and the destroyed aneurysm wall, causing the devastating rupture. The major difference between this aneurysm and the one with similar size, location and morphology was the lack of reduction in intra-aneurysmal flow velocities, which may explain the devastating effect of a persisting high energy inflow jet.

In the 5-mm aneurysm demonstrating persistent patency during 12 months after treatment, mean and maximal velocities as well as WSS were all significantly reduced according to the simulations; however, the flow pattern remained the same (Fig. 2). We hypothesise that with an unchanged, regular, single-vortex flow pattern, the induced velocity and WSS reductions probably still did not reach the threshold of thrombosis at this aneurysm size, keeping the aneurysm patent. In a similar size and shape aneurysm with a similar AR (case no. 1), despite less important reduction rates in intra-aneurysmal velocity and WSS, this aneurysm showed complete occlusion and reverse remodelling during the follow up. Besides probable individual differences in the haemodynamic conditions, the major difference between the two cases was that in the case with thrombosis the FD 
induced a significant change in the intra-aneurysmal flow patterns, which may be an explanation for its thrombosis.

Interestingly, the FD induced a significant change in flow pattern only in one of the eight aneurysms. In this case, besides a significant reduction in flow velocities and WSS, the streamlines of the flow were also dramatically changed, with disappearance of the jet type inflow (Fig. 1). If we consider the term "diversion", with the meaning of an instance of turning something aside from its natural course, the real flow diversion effect was achieved only in this case, while in the vast majority of the aneurysms at this location, only a magnitude reduction of the flow energy was induced by the device. In five of these seven aneurysms, this reduction was enough to induce thrombosis and complete occlusion. Despite significant velocity and WSS reductions, one aneurysm remained patent for 12 months after treatment. Another aneurysm, where the FD did not modify the very strong inflow jet, ruptured 5 days after treatment. These facts may suggest that, in certain cases, modification of flow patterns or, more importantly, reductions of velocity and WSS may be necessary. This could be achieved by applying multiple overlapping FD layers or by intra-saccular aneurysm coiling.

The limitations of the study are related to: the low number of cases analysed; the difference in size and morphological characteristics of the involved aneurysms; the lack of patient specific flow profile data, with all cases having applied the same flow profile; the idealised deployment of the FD device in the CFD simulations, which may not always be the case in the clinical setting; the lack of high frame rate angiographic imaging before and after FD deployment to be correlated with the CFD simulations for validation reasons. With the new angiographic devices this option is becoming more widely available in the near future.

The power of this study does not yet allow us to predict how many FD layers would suffice to induce controlled thrombosis and reverse remodelling of a specific aneurysm. We believe, however, that CFD simulations may now be more regularly applied prior to treatment in all elective cases and, by gathering more data with clinical correlation, may become an additional tool in treatment planning.

\section{Conclusions}

In this series of para-ophthalmic aneurysms treated by flow diversion obtained by endovascular deployment of a singlelayer, braided, low-porosity, high-pore-density FD device, we correlated clinical occlusion rates with retrospective CFD assessments. We found main flow modification to consist in mean intra-aneurismal blood flow velocity and WSS reductions. These changes may be enough to induce complete thrombosis and subsequent aneurysm reverse remodelling as clinically observed in the majority of the studied cases; however, exact threshold values of flow reduction could not be determined, since all aneurysms have their characteristic flow profile. Modification of the flow pattern was observed in only one of eight cases and we therefore cannot comment on a possible predictive role of this parameter. Further, as observed, a single-layer FD may not be able to break a very strong inflow jet at the aneurysm entry point but still reduce the mean intra-aneurysmal flow, and by this fail to clot at all or induce partial thrombosis only. Such thrombosis may have a destructive effect on the aneurysm wall, which - in combination with a persisting inflow jet - may cause the aneurysm to rupture with delay. We believe that these mechanisms could explain retrospectively the two treatment failures observed in the cases evaluated. Numerical simulation may possibly help to predict such failures and, if proven by larger series, become a tool to provide a safer treatment to our patients.

Finally, in lack of flow pattern changes one might challenge the currently common used term of "flow diverter" for this type of vascular repair devices.

Acknowledgments This work was partially supported by a grant from the Swiss National Science Foundation (SNSF) (CR32I3127008). The CFD studies were partially financed by Balt International.

Conflicts of interest Z. Kulcsar and I. Wanke are contractual proctors for Silk FD implantation with Balt Int.

\section{References}

1. Aird WC (2007) Vascular bed-specific thrombosis. J Thromb Haemost 5(Suppl 1):283-291

2. Augsburger L, Reymond P, Rufenacht DA, Stergiopulos N (2011) Intracranial stents being modeled as a porous medium: flow simulation in stented cerebral aneurysms. Ann Biomed Eng 39 (2):850-863

3. Byrne JV, Beltechi R, Yarnold JA, Birks J, Kamran M (2010) Early experience in the treatment of intra-cranial aneurysms by endovascular flow diversion: a multicentre prospective study. PLoS One 5 (9).pii: e12492

4. Cebral JR, Mut F, Raschi M, Scrivano E, Ceratto R, Lylyk P, Putman CM (2011) Aneurysm rupture following treatment with flow-diverting stents: computational hemodynamics analysis of treatment. AJNR Am J Neuroradiol 32(1):27-33

5. Frösen J, Piippo A, Paetau A, Kangasniemi M, Niemelä M, Hernesniemi J, Jääskeläinen J (2004) Remodeling of saccular cerebral artery aneurysm wall is associated with rupture: histological analysis of 24 unruptured and 42 ruptured cases. Stroke 35(10):2287-2293

6. Kataoka K, Taneda M, Asai T, Kinoshita A, Ito M, Kuroda R (1999) Structural fragility and inflammatory response of ruptured cerebral aneurysms. A comparative study between ruptured and unruptured cerebral aneurysms. Stroke 30(7):1396-1401

7. Kulcsár Z, Ernemann U, Wetzel SG, Bock A, Goericke S, Panagiotopoulos V, Forsting M, Ruefenacht DA, Wanke I (2010) High-profile flow diverter (silk) implantation in the 
basilar artery: efficacy in the treatment of aneurysms and the role of the perforators. Stroke 41(8):1690-1696

8. Kulcsár Z, Houdart E, Bonafé A, Parker G, Millar J, Goddard AJ, Renowden S, Gál G, Turowski B, Mitchell K, Gray F, Rodriguez M, van den Berg R, Gruber A, Desal H, Wanke I, Rüfenacht DA (2011) Intra-aneurysmal thrombosis as a possible cause of delayed aneurysm rupture after flow-diversion treatment. AJNR Am J Neuroradiol 32(1):20-25

9. Lylyk P, Miranda C, Ceratto R, Ferrario A, Scrivano E, Luna HR, Berez AL, Tran Q, Nelson PK, Fiorella D (2009) Curative endovascular reconstruction of cerebral aneurysms with the pipeline embolization device: the Buenos Aires experience. Neurosurgery 64(4):632-642, discussion 642-3; quiz N6

10. Nelson PK, Lylyk P, Szikora I, Wetzel SG, Wanke I, Fiorella D (2011) The pipeline embolization device for the intracranial treatment of aneurysms trial. AJNR Am J Neuroradiol 32(1):34-40

11. Reymond P, Merenda F, Perren F, Rüfenacht D, Stergiopulos N (2009) Validation of a one-dimensional model of the systemic arterial tree. Am J Physiol Heart Circ Physiol 297(1):H208-H222
12. Sadasivan C, Cesar L, Seong J, Rakian A, Hao Q, Tio FO, Wakhloo AK, Lieber BB (2009) An original flow diversion device for the treatment of intracranial aneurysms: evaluation in the rabbit elastase-induced model. Stroke 40(3):952-958

13. Szikora I, Berentei Z, Kulcsar Z, Marosfoi M, Vajda ZS, Lee W, Berez A, Nelson PK (2010) Treatment of intracranial aneurysms by functional reconstruction of the parent artery: the Budapest experience with the pipeline embolization device. AJNR Am J Neuroradiol 31(6):1139-1147

14. Ujiie H, Tamano Y, Sasaki K, Hori T (2001) Is the aspect ratio a reliable index for predicting the rupture of a saccular aneurysm? Neurosurgery 48(3):495-502, discussion 502-3

15. Wiebers DO, Whisnant JP, Huston J, Meissner I, Brown RD, Piepgras DG, Forbes GS, Thielen K, Nichols D, O'Fallon WM, Peacock J, Jaeger L, Kassell NF, Kongable-Beckman GL, Torner JC, International Study of Unruptured Intracranial Aneurysms Investigators (2003) Unruptured intracranial aneurysms: natural history, clinical outcome, and risks of surgical and endovascular treatment. Lancet 362(9378):103-110 